\title{
Optimization methods for achieving high diffraction efficiency with perfect electric conducting gratings
}

\author{
Rubén Aylwin ${ }^{1}$, Gerardo Silva-Oelker ${ }^{2}$, Carlos Jerez-Hanckes ${ }^{3}$, and Patrick Fay ${ }^{4}$ \\ ${ }^{1}$ Department of Electrical Engineering, Pontificia Universidad Católica de Chile, Santiago, Chile. \\ ${ }^{2}$ Department of Mechanical Engineering, Universidad Tecnológica Metropolitana, Santiago, Chile. \\ ${ }^{3}$ Faculty of Engineering and Sciences, Universidad Adolfo Ibáñez, Santiago, Chile. \\ ${ }^{4}$ Department of Electrical Engineering, University of Notre Dame, IN, USA.
}

November 4, 2020

\begin{abstract}
This work presents the implementation, numerical examples and experimental convergence study of first- and second-order optimization methods applied to one-dimensional periodic gratings. Through boundary integral equations and shape derivatives, the profile of a grating is optimized such that it maximizes the diffraction efficiency for given diffraction modes for transverse electric polarization. We provide a thorough comparison of three different optimization methods: a first-order method (gradient descent); a second-order approach based on a Newton iteration, where the usual Newton step is replaced by taking the absolute value of the eigenvalues given by the spectral decomposition of the Hessian matrix to deal with non-convexity; and the Broyden-Fletcher-Goldfarb-Shanno (BFGS) algorithm, a quasi-Newton method. Numerical examples are provided to validate our claims. Moreover, two grating profiles are designed for high efficiency in the Littrow configuration and then compared to a high efficiency commercial grating. Conclusions and recommendations, derived from the numerical experiments, are provided as well as future research avenues.
\end{abstract}

\section{Introduction}

One-dimensional gratings are able to diffract, split, reflect and transmit light, depending on geometrical parameters such as amplitude, period, and shape. For example, it is well known that metallic gratings exhibit Wood's anomalies [64, 47] and cavity resonances [46]. Of particular interest in applications is the diffraction efficiency - defined as the amount of power diffracted in one mode [44]. Due to these properties, gratings have several applications in science and engineering, ranging from X-ray spectroscopy [37, energy conversion devices such as photovoltaics [58] and thermophotovoltaics [56], beam splitters [16], quantum cascade lasers [17], and filters [40, to name a few. Consequently, a large body of literature has been devoted to the study of physical phenomena [33, 24, 36], mathematical modeling [11, 67, 28, 55], fabrication [35, 25, 51], and optimization of gratings.

Among the optimization methods for designing gratings, genetic algorithms and particle swarm optimization have been studied and implemented for applications such as energy conversion [54, 12] and filters [53]. These methods have shown to be practical options due to their simplicity and flexibility in implementation. However, the aforementioned techniques suffer from disadvantages such as partial convergence and the need for a large number of evaluations of the cost function. In 
contrast, methods that rely on following the gradient direction to minimize a suitable cost function can be more appropriate for some problems. Roger [49], for instance, studied the optimization of a perfect electric conducting (PEC) grating by the steepest-descent and conjugate-gradient methods along with boundary integral equations (BIEs). Recently, Bao et al. [6] implemented the Landweber iteration also along with BIE for inverse problems, showing the applicability of gradient optimization. Methods based on so-called shape derivatives have been theoretically proposed [19, 20] for optimization and successfully applied to the design of devices [41. However, grating geometry optimization using shape derivatives, to the best of our knowledge, has not been explored yet. This work aims to provide details and examples of first- and second-order methods based on shape gradients for PEC grating profile optimization.

The use of optimization algorithms can lead to non-trivial grating shapes (e.g., échelle or holographic). Fortunately, mainstream fabrication approaches for manufacturing complex profiles have become available, including electron beam lithography [66, 60, laser ablation [4], deposition-andetch based approaches [62, 2], and interference lithography [29, 22. While not as well developed commercially, intricate structures can also be fabricated using techniques such as nanoimprint [65. 27, surface wrinkling [63, and scanning-probe lithography [32]. With these tools, complex grating structures can now be manufactured, thereby increasing the range of shape possibilities and making it important to design and fabricate optimal gratings.

In this work, we implement and compare first- and second-order optimization algorithms to maximize the diffraction efficiency for transverse electric polarization. Diffraction efficiency was chosen as the target figure of merit for its importance in applications [10. In our approach, the wave scattering model problem in computational volume is reduced to one defined on the grating boundary through an integral formulation and a suitable quasi-periodic Green's function. Optimization is carried out by minimizing or maximizing a cost or objective function using firstand second-order shape gradients [13] of the defined far-field operator, which maps the grating profile to the diffraction field components. In the case of the first-order method, it is shown that the shape derivative approach is equivalent to the work presented by Roger [49. However, since this optimization problem can be highly non-convex, additional techniques are explored to improve performance. In this regard, two Newton-based second-order algorithms are implemented and studied. First, a second-order method with a modified step [15, 43], able to deal with non-convexity, is considered. Secondly, the quasi-Newton Broyden-Fletcher-Goldfarb-Shanno (BFGS) algorithm is implemented for comparison. Numerical results show that, for certain objective functions, the modified Newton method converges quadratically, whereas the BFGS algorithm converges superlinearly. Moreover, these methods reduce both computational time and the number of iterations required to find a maximum. These results pave the way for more efficient approaches of grating optimization and periodic structures in general. Furthermore, the studied techniques can also be applied to inverse design problems.

The rest of the paper is organized as follows. In Section 2 , the wave scattering by a grating problem is presented, along with the boundary integral formulation and the definition of diffraction efficiency. Section 3 describes the optimization methods: shape derivatives are introduced and firstand second-order methods are detailed. Numerical examples are provided in Section 4, analyzing computation times as well as designing grating profiles. Finally, conclusions are drawn in Section 5. 


\section{Scattering Problem and Boundary Integral Formulation}

A PEC grating with period $\Lambda$ and surface given by $\widetilde{\Gamma} \times \mathbb{R}$ is considered (see Fig. 1), where $\widetilde{\Gamma}$ is assumed to be (at least) Lipschitz continuous. The domain $\widetilde{D} \times \mathbb{R} \subset \mathbb{R}^{3}$ is defined as the open region of propagation above the grating surface, assumed to be free space and characterized by its impedance $\eta=\sqrt{\mu / \varepsilon}$, where $\varepsilon$ and $\mu$ correspond to vacuum permittivity and permeability, respectively.

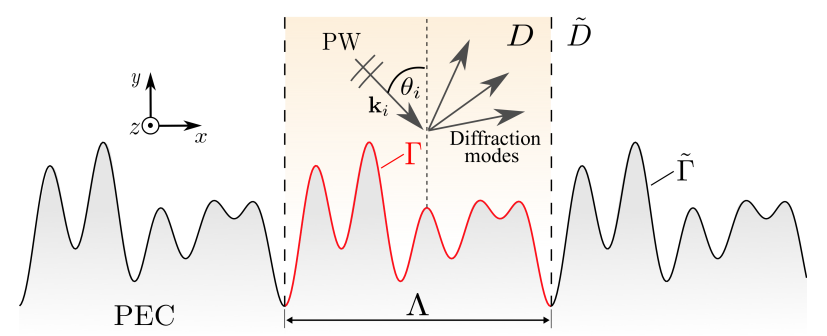

Figure 1: Perfectly conducting grating with a period $\Lambda$ : the domain $\widetilde{D}$ above the grating and its surface $\widetilde{\Gamma}$ are shown. Both $D$ and $\Gamma$ are defined in one period.

\subsection{Scattering Problem}

We consider the scattering of a monochromatic plane wave (PW) with wave vector $\mathbf{k}_{i}$ by a grating surface described in Fig. 1. Furthermore, we assume the wave vector to be such that it has no $z$ component $\left(\mathbf{k}_{i}=\left(k_{x}, k_{y}\right)\right)$, so that Maxwell's equations may be decomposed into transverse electric (TE) and transverse magnetic (TM) polarizations (see [7] and references therein). For brevity, only TE polarization $\left(E_{x}=E_{y}=H_{z}=0\right)$ is considered in this study. Nevertheless, optimization for TM polarization follows the same approach with different boundary conditions. Hence, this work focuses on the $z$ component of the electric field, defined as:

$$
u(\mathbf{r})=E_{z}(\mathbf{r}) \quad \mathbf{r} \in \widetilde{D}
$$

where $\mathbf{r}:=(x, y) \in \mathbb{R}^{2}$ is the position vector. Then, the total field $u$ satisfies the Helmholtz equation:

$$
\begin{aligned}
\Delta u+k^{2} u=0 & \text { in } \quad \widetilde{D}, \\
u=0 & \text { on } \quad \widetilde{\Gamma},
\end{aligned}
$$

where $k:=\omega / c$ is the wavenumber. As previously stated, we consider the scattering of an incident PW (see Fig. 11):

$$
u^{i}(\mathbf{r}):=E_{0} e^{\imath \mathbf{k}_{i} \cdot \mathbf{r}} \quad \forall \mathbf{r} \in \widetilde{D},
$$

onto the grating surface $\widetilde{\Gamma}$, where $\mathbf{k}_{i}=\left(k_{x}, k_{y}\right)=k\left(\sin \theta_{i},-\cos \theta_{i}\right)$ is the incident wave vector with incidence angle $\theta_{i}$ and null $z$ component, and $E_{0}>0$ corresponds to the incident electric field amplitude. By linearity, the total field is $u=u^{i}+u^{s}$, where $u^{s}$ is the scattered field. Since PWs satisfy (2), we may restate the previous system as a problem for the scattered field as follows:

$$
\begin{aligned}
\Delta u^{s}+k^{2} u^{s} & =0 \quad \text { in } \widetilde{D}, \\
u^{s} & =-u^{i} \quad \text { on } \widetilde{\Gamma} .
\end{aligned}
$$


The grating periodicity and the quasi-periodicity of $u^{i}$, i.e.

$$
u^{i}(x+n \Lambda, y)=u^{i}(x, y) e^{\imath k_{x} \Lambda n} \quad \forall n \in \mathbb{Z},
$$

enable us to restate the problem for the scattered field on one cell $D$-bounded in the $x$-directionof the infinite periodic domain $\widetilde{D}$ by enforcing the condition in (6) at the left and right boundaries of $D$. However, the problem still lacks appropriate radiation conditions at infinity, which, for the sake of brevity, will not be derived here. Instead, we refer to [7, 38, 30] and references therein for a derivation and discussion of adequate radiation conditions in periodic problems. For a fixed $d \in \mathbb{R}$, such that $\widetilde{\Gamma}$ lies strictly below the line $y=d$, we define the following domains:

$$
D:=\{\mathbf{r} \in \widetilde{D}: x \in(0, \Lambda)\} \quad \text { and } \quad D_{d}:=\{\mathbf{r} \in D: y<d\} .
$$

Analogously, we define the grating surface over one period

$$
\Gamma:=\{\mathbf{r} \in \widetilde{\Gamma}: x \in(0, \Lambda)\} .
$$

Additionally, the Sobolev space of square integrable functions with square integrable first-order derivatives on $D_{d}$ that satisfy (6) is denoted $H_{k_{x} ; \Lambda}^{1}\left(D_{d}\right)$. We then seek to find a field $u^{s} \in H_{k_{x} ; \Lambda}^{1}\left(D_{d}\right)$ that solves

$$
\begin{aligned}
\Delta u^{s}+k^{2} u^{s} & =0 \quad \text { in } D_{d}, \\
u^{s} & =-u^{i}=: g \quad \text { on } \Gamma, \\
u^{s}(x, y) & =\sum_{n \in \mathbb{Z}} u_{n}(d) e^{\imath\left(k_{y, n}(y-d)+k_{x, n} x\right)} \quad \forall y \geq d,
\end{aligned}
$$

where, for each $n \in \mathbb{Z}, u_{n}(d)$ are the Rayleigh coefficients, $k_{x, n}:=k \sin \theta_{i}+2 \pi n / \Lambda$, and

$$
k_{y, n}:=\left\{\begin{array}{lll}
\sqrt{k^{2}-k_{x, n}^{2}} & \text { if } \quad k^{2} \geq k_{x, n}^{2} \\
\imath \sqrt{k_{x, n}^{2}-k^{2}} & \text { if } \quad k^{2}<k_{x, n}^{2}
\end{array} .\right.
$$

The last line in (77) corresponds to the radiation condition for the periodic domain, also known as the Rayleigh expansion for $u^{s}$ [7, 44, 30]. In order to avoid Rayleigh anomalies, we further assume that no $n \in \mathbb{Z}$ exists such that $k_{x, n}^{2}=k^{2}$.

\subsection{Boundary Integral Equations}

To find the scattered field, we use BIEs - particularly the electric field integral equation - motivated by the unboundedness of $D$. Therefore, we make the following representation Ansatz:

$$
u^{s}(\mathbf{r})=(\mathrm{SL} j)(\mathbf{r}), \quad \mathbf{r} \in D,
$$

where $j$ is an unknown surface density (in this case, the surface current density) and SL is the quasi-periodic single-layer operator, whose action on the density $j$ may be represented through the quasi periodic Green's function $G^{p}$ as:

$$
(\mathrm{SL} j)(\mathbf{r}):=\imath k \eta \int_{\Gamma} G^{p}\left(\mathbf{r}, \mathbf{r}^{\prime}\right) j\left(\mathbf{r}^{\prime}\right) d \Gamma\left(\mathbf{r}^{\prime}\right), \quad \mathbf{r} \in D .
$$

Using (8), (9), and the boundary conditions in (7), the BIE for the unknown surface density $j$ may be written as:

$$
\mathcal{V} j=-u^{i} \text { on } \Gamma
$$


where $\mathcal{V}$ is the boundary integral operator mapping the surface density $j$ to the boundary values of SL $j$. The quasi-periodic Green's function, $G^{p}$, is given by [34, 61]:

$$
G^{p}\left(\mathbf{r}, \mathbf{r}^{\prime}\right)=\frac{\imath}{2 \Lambda} \sum_{n=-\infty}^{\infty} \frac{e^{\imath k_{x, n}\left(x-x^{\prime}\right)+\imath k_{y, n}\left|y-y^{\prime}\right|}}{k_{y, n}}
$$

\subsection{The Scattered Far-Field}

As displayed in (7), the scattered field may be decomposed as a linear combination of complex exponentials. Of these, only those corresponding to $n \in \mathbb{Z}$ such that $k^{2}>k_{x, n}^{2}$ do not decay as $y$ grows to infinity - as noted above, we assume no $n \in \mathbb{Z}$ exists such that $k^{2}=k_{x, n}^{2}$. Therefore, the scattered far-field - the scattered field for $d$ far from the grating surface - depends solely on the Rayleigh coefficients corresponding to a real value for $k_{y, n}$, i.e., on $\left\{u_{n}(d)\right\}_{n \in \mathcal{N}\left(k, \theta_{i}, \Lambda\right)}$ where:

$$
\mathcal{N}\left(k, \theta_{i}, \Lambda\right):=\left\{n \in \mathbb{Z}: k>\left|k \sin \left(\theta_{i}\right)+2 \pi n / \Lambda\right|\right\} .
$$

The coefficients of the Rayleigh expansion in the radiation condition of Eq. (7) may be extracted from the scattered field as follows:

$$
\begin{aligned}
u_{n}(d) & =\frac{1}{\Lambda} \int_{0}^{\Lambda} u^{s}(x, d) e^{-\imath k_{x, n} x} d x \\
& =-e^{\imath k_{y, n} d} \frac{k \eta}{2 \Lambda k_{y, n}} \int_{\Gamma} j(\mathbf{r}) e^{-\imath \mathbf{K}_{n} \cdot \mathbf{r}} d \Gamma(\mathbf{r}),
\end{aligned}
$$

where $\mathbf{K}_{n}:=\left(k_{x . n}, k_{y, n}\right)$. Then, the associated grating diffraction efficiency can be computed

through $e_{n}:=\frac{k_{y, n}}{k_{y}}\left|u_{n}(d)\right|^{2}$, further justifying the choice to employ BIE to solve the scattering problem. Henceforth, we omit the dependency of the coefficients $u_{n}$ on $d$, since it is of no significance for the computation of the efficiencies, as indicated by (12) (the magnitude $-u_{n}(d) e^{-\imath k_{y, n} d}$ is constant in $d$ ). Coefficients $u_{n}$ are thus calculated using

$$
u_{n}:=\frac{k \eta}{2 \Lambda k_{y, n}} \int_{\Gamma} j(\mathbf{r}) e^{-\imath \mathbf{K}_{n} \cdot \mathbf{r}} d \Gamma(\mathbf{r}) .
$$

\section{Optimization}

We aim to find optimal grating profiles in the sense that they maximize (or minimize) functions of the diffraction efficiencies, e.g.,

$$
\max _{\Gamma} e_{n}(\Gamma), \quad \min _{\Gamma} \sum_{n \in \mathcal{N}^{\prime}}\left(e_{n}(\Gamma)-e_{n}^{\mathrm{obj}}\right)^{2}
$$

for some $\mathcal{N}^{\prime} \subseteq \mathcal{N}\left(k, \theta_{i}, \Lambda\right)$ and some objective efficiencies $e_{n}^{\text {obj }}$ in the case of minimization. With this in mind, we turn to the problem of computing derivatives of the far-field Rayleigh coefficients as functions of the grating geometry for the implementation of local search optimization algorithms. To do so, we introduce the $n$-th mode far-field operator as

$$
\mathcal{F}_{n}: \Gamma \mapsto u_{n}
$$

This operator maps a grating profile $\Gamma$ to $\mathcal{F}_{n}(\Gamma)=u_{n}$, the $n$-th coefficient of the Rayleigh expansion of the diffracted field. Henceforth, we assume $k, \Lambda$, and $\theta_{i}$ to be fixed. 


\subsection{Shape Calculus and Shape Derivatives}

To compute derivatives of the far-field operator with respect to the grating geometry, we use tools from shape calculus [45, 57]. For $m \in \mathbb{N}$ and a given grating profile $\Gamma$ of class $C^{m}$ (the $m$-th derivative exists and is continuous) we introduce the following space of periodic functions:

$$
C_{p}^{\ell}(\Gamma):=\left\{\tau \in C^{\ell}\left(\Gamma ; \mathbb{R}^{2}\right): \tau \text { is periodic }\right\}
$$

for any non-negative integer $\ell \leq m$. Moreover, for any $a \in C_{p}^{1}(\Gamma)$, we introduce the perturbed grating profile as

$$
\Gamma_{a}:=\{\mathbf{r}+a(\mathbf{r}): \mathbf{r} \in \Gamma\},
$$

whenever $a$ is small enough so that $\Gamma_{a}$ does not self intersect. Then, the definition of the shape derivative of $\mathcal{F}_{n}$ at $\Gamma$ in the direction $a$ is

$$
\mathcal{F}_{n}^{\prime}(\Gamma ; a):=\lim _{\epsilon \rightarrow 0} \frac{1}{\epsilon}\left(\mathcal{F}_{n}\left(\Gamma_{\epsilon a}\right)-\mathcal{F}_{n}(\Gamma)\right),
$$

whenever the limit in the right-hand side of (15) exists [31, 30, 45, 57. Moreover, we say $\mathcal{F}_{n}$ is shape-differentiable on $C_{p}^{\ell}(\Gamma)$ if $\mathcal{F}_{n}^{\prime}(\Gamma ; \cdot)$ is a linear and continuous functional on $C_{p}^{\ell}(\Gamma)$. The computation of $\mathcal{F}_{n}^{\prime}(\Gamma ; a)$, for $a$ in $C_{p}^{2}(\Gamma)$, can be performed by solving a problem analogous to (77) through the use of the following relation:

$$
\mathcal{F}_{n}^{\prime}(\Gamma ; a)=\mathcal{F}_{n}\left(u^{\prime}[a]\right),
$$

where $u^{\prime}[a]$ is the unique solution in $H_{k_{x} ; \Lambda}^{1}\left(D_{d}\right)$ to the following scattering problem:

$$
\begin{gathered}
\Delta u^{\prime}[a]+k^{2} u^{\prime}[a]=0 \quad \text { in } D_{d}, \\
u^{\prime}[a]=-(a \cdot \nu) \frac{\partial}{\partial \nu} u \quad \text { on } \Gamma, \\
u^{\prime}[a](\mathbf{r})=\sum_{n \in \mathbb{Z}} u_{n}^{\prime}[a](d) e^{\imath\left(k_{y, n}(y-d)+k_{x, n} x\right)} \quad \forall y \geq d,
\end{gathered}
$$

where $\nu$ is the normal vector to $\Gamma$. This result follows by modifying the proof of Theorem 2.1 in [31] to the quasi-periodic setting ( $c f .[3])$.

The second-order shape derivative of $\mathcal{F}_{n}$ at $\Gamma$ along the directions $a_{1}, a_{2} \in C_{p}^{1}(\Gamma)$ is defined as

$$
\mathcal{F}_{n}^{\prime \prime}\left(\Gamma ; a_{1} ; a_{2}\right):=\lim _{\epsilon \rightarrow 0} \frac{1}{\epsilon}\left(\mathcal{F}_{n}^{\prime}\left(\Gamma_{\epsilon a_{2}} ; a_{1} \circ \psi_{\epsilon}\right)-\mathcal{F}_{n}^{\prime}\left(\Gamma ; a_{1}\right)\right),
$$

with

$$
\psi_{\epsilon}:=\phi_{\epsilon}^{-1}, \quad \phi_{\epsilon}:=\left\{\begin{array}{ll}
\Gamma & \rightarrow \Gamma_{\epsilon a_{2}} \\
\mathbf{r} & \mapsto \mathbf{r}+\epsilon a_{2}(\mathbf{r})
\end{array} .\right.
$$

This definition was introduced in the context of scattering from bounded obstacles in [26] and satisfies $\mathcal{F}_{n}^{\prime \prime}\left(\Gamma ; a_{1} ; a_{2}\right)=\mathcal{F}_{n}^{\prime \prime}\left(\Gamma ; a_{2} ; a_{1}\right)$, i.e., it is symmetric. Analogous to the case of first-order shape derivatives, the computation of $\mathcal{F}_{n}^{\prime \prime}\left(\Gamma ; a_{1} ; a_{2}\right)$ for $a_{1}$ and $a_{2}$ in $C_{p}^{3}(\Gamma)$ follows from:

$$
\mathcal{F}_{n}^{\prime \prime}\left(\Gamma ; a_{1} ; a_{2}\right)=\mathcal{F}_{n}\left(u^{\prime \prime}\left[a_{1} ; a_{2}\right]\right)
$$


where $u^{\prime \prime}\left[a_{1} ; a_{2}\right]$ is the unique solution in $H_{k_{x} ; \Lambda}^{1}\left(D_{d}\right)$ to the following scattering problem:

$$
\begin{gathered}
\Delta u^{\prime \prime}\left[a_{1}, a_{2}\right]+k^{2} u^{\prime \prime}\left[a_{1}, a_{2}\right]=0 \quad \text { in } D_{d}, \\
u^{\prime \prime}\left[a_{1}, a_{2}\right]=-\left(a_{1} \cdot \nu\right) \frac{\partial u^{\prime}\left[a_{2}\right]}{\partial \nu}-\left(a_{2} \cdot \nu\right) \frac{\partial u^{\prime}\left[a_{1}\right]}{\partial \nu} \\
+\left(\left(a_{1} \cdot \nu\right)\left(a_{2} \cdot \nu\right)-\left(a_{1} \cdot \tau\right)\left(a_{2} \cdot \tau\right)\right) \kappa \frac{\partial u}{\partial \nu} \\
+\left(\left(a_{1} \cdot \tau\right)\left(\tau \cdot \nabla\left(a_{2} \cdot \nu\right)\right)+\left(a_{2} \cdot \tau\right)\left(\tau \cdot \nabla\left(a_{1} \cdot \nu\right)\right)\right) \frac{\partial u}{\partial \nu} \quad \text { on } \Gamma, \\
u^{\prime \prime}\left[a_{1}, a_{2}\right](\mathbf{r})=\sum_{n \in \mathbb{Z}} u_{n}^{\prime \prime}\left[a_{1} ; a_{2}\right](d) e^{\imath\left(k_{y, n}(y-d)+k_{x, n} x\right)} \quad \forall y \geq d,
\end{gathered}
$$

where $\kappa$ denotes the curvature of $\Gamma$, and $\nu$ and $\tau$ denote the normal and tangent vectors to the surface, respectively.

Throughout the following sections, we consider, without loss of generality, $\Lambda \equiv 1$. Moreover, recalling $\mathbf{r}=(x, y) \in \mathbb{R}^{2}$ we assume $\Gamma$ to be given as follows

$$
\Gamma:=\left\{\mathbf{r}: y=\sum_{\ell=1}^{N} \mathcal{A}_{\ell} \sin (2 \pi \ell x)+\mathcal{B}_{\ell} \cos (2 \pi \ell x), x \in(0,1)\right\},
$$

thus fulfilling the conditions that allow us to compute first and second-order shape derivatives as solutions to the aforementioned boundary value problems. Then, $\mathcal{F}_{n}(\Gamma)=\mathcal{F}_{n}\left(\mathcal{A}_{1}, \mathcal{B}_{1}, \ldots, \mathcal{A}_{N}, \mathcal{B}_{N}\right)$ and the optimization problem is now finite-dimensional over the $2 N$ variables $\left\{\mathcal{A}_{\ell}\right\}_{\ell=1}^{N}$ and $\left\{\mathcal{B}_{\ell}\right\}_{\ell=1}^{N}$. It holds that

$$
\frac{\partial \mathcal{F}_{n}}{\partial \mathcal{A}_{\ell}}=\mathcal{F}_{n}^{\prime}\left(\Gamma ; \mathrm{e}_{y} \sin (2 \pi \ell x)\right) \quad \text { and } \quad \frac{\partial \mathcal{F}_{n}}{\partial \mathcal{B}_{\ell}}=\mathcal{F}_{n}^{\prime}\left(\Gamma ; \mathrm{e}_{y} \cos (2 \pi \ell x)\right)
$$

where $\mathrm{e}_{y}$ is the canonical vector in the $y$-direction. Note that $\mathrm{e}_{y} \sin (2 \pi \ell x)$ and $\mathrm{e}_{y} \cos (2 \pi \ell x)$ represent directions, i.e., we need only consider perturbations of the form $a(\boldsymbol{r})=\mathrm{e}_{y} a_{y}(\boldsymbol{r})$, with $a_{y}$ scalar. Moreover, our choice of $\Gamma$ implies that $2 N$ problems are solved to compute every required derivative. Analogous relations can be found for the second-order derivatives of $\mathcal{F}_{n}$.

\subsection{Adjoint Method for the Computation of Shape Derivatives}

The computation of each derivative of $\mathcal{F}_{n}\left(\mathcal{A}_{1}, \mathcal{B}_{1}, \ldots, \mathcal{A}_{N}, \mathcal{B}_{N}\right)$ requires solving a BIE with a varying right-hand side. The same holds for each second-order derivative, which also requires the computation of boundary data of first-order shape derivatives. We may achieve the computation of derivatives more efficiently through the adjoint method [59].

As noted previously, $j$ is the solution to (10), which also satisfies $j=\frac{\partial}{\partial \nu}\left(u^{s}+u^{i}\right)$. Let us also introduce $\tilde{j}$ as a surface density such that $u^{\prime}[a](\mathbf{r})=(\mathrm{SL} \tilde{j})(\mathbf{r})$ for $\mathbf{r}$ in $D$. Since the shape derivative, $u^{\prime}[a]$, satisfies (16), then $\tilde{j}$ solves a BIE similar to (10), but considering the boundary condition in (16), i.e., $\mathcal{V} \tilde{j}=-(a \cdot \nu) j$ on $\Gamma$. Recognizing that the coefficients $u_{n}$ in (13) can be written as a duality product and denoting $g_{n}(\mathbf{r}):=e^{-i \mathbf{K}_{n} \cdot \mathbf{r}}$, we obtain for $a \in C_{p}^{2}(\Gamma)$ :

$$
\begin{aligned}
\mathcal{F}_{n}^{\prime}(\Gamma ; a) & =\frac{k \eta}{2 \Lambda k_{y, n}}\left\langle\tilde{j}, g_{n}\right\rangle_{\Gamma}=\frac{k \eta}{2 \Lambda k_{y, n}}\left\langle\mathcal{V}^{-1}(-(a \cdot \nu) j), g_{n}\right\rangle_{\Gamma} \\
& =\frac{k \eta}{2 \Lambda k_{y, n}}\left\langle\mathcal{V}^{-\top}\left(g_{n}\right),-(a \cdot \nu) j\right\rangle_{\Gamma} .
\end{aligned}
$$


Hence, if $j^{\text {adj }}$ is a surface density that solves the BIE:

$$
\mathcal{V}^{\top} j^{\text {adj }}=g_{n},
$$

then, we may compute shape derivatives in the direction $a \in C_{p}^{2}(\Gamma)$ as:

$$
\mathcal{F}_{n}^{\prime}(\Gamma ; a)=\frac{k \eta}{2 \Lambda k_{y, n}}\left\langle j^{\text {adj }},-(a \cdot \nu) j\right\rangle_{\Gamma}
$$

According to (19), all of the first-order derivatives can be computed by solving two BIEs of the form of (10) and then computing the specified integral. In (18), $\mathcal{V}^{\top}$ is the boundary integral operator analogous to $\mathcal{V}$ for the adjoint quasi-periodic Green's function given by

$$
G_{\text {adj }}^{p}\left(\mathbf{r}, \mathbf{r}^{\prime}\right)=\frac{\imath}{2 \Lambda} \sum_{n=-\infty}^{\infty} \frac{e^{\imath \widetilde{k}_{x, n}\left(x-x^{\prime}\right)+\imath k_{y, n}\left|y-y^{\prime}\right|}}{k_{y, n}},
$$

where $\widetilde{k}_{x, n}:=-k \sin \left(\theta_{i}\right)+n$ for all $n \in \mathbb{N}$. The same formula for the first-order shape derivatives of the far-field operator was found in [48] through the use of reciprocity relations between solutions of scattering problems. Analogously,

$$
\mathcal{F}_{n}^{\prime \prime}\left(\Gamma ; a_{1} ; a_{2}\right)=\frac{k \eta}{2 \Lambda k_{y, n}}\left\langle j^{\text {adj }},\left.u^{\prime \prime}\left[a_{1}, a_{2}\right]\right|_{\Gamma}\right\rangle_{\Gamma} .
$$

\subsection{First-Order Optimization}

Throughout this and the following two subsections, we consider the optimization problem of minimizing a function $f: \mathbb{R}^{2 N} \rightarrow \mathbb{R}$ such that

$$
f\left(\mathcal{A}_{1}, \mathcal{B}_{1}, \ldots, \mathcal{A}_{N}, \mathcal{B}_{N}\right)=\tilde{f}\left(e_{n}\left(\mathcal{A}_{1}, \mathcal{B}_{1}, \ldots, \mathcal{A}_{N}, \mathcal{B}_{N}\right)\right)
$$

for a smooth function $\tilde{f}: \mathbb{R} \rightarrow \mathbb{R}$ (e.g., $\tilde{f}(x)=x, \tilde{f}(x)=-x$ or $\tilde{f}(x)=(x-c)^{2}$ for some $\left.c \in \mathbb{R}\right)$. Hence, our optimization problem may be stated as

$$
\min _{\mathbf{x} \in \mathcal{O}_{\mathrm{ad}}} f(\mathbf{x})=\min _{\Gamma \in \mathcal{O}_{\mathrm{ad}}^{S}} \tilde{f}\left(e_{n}(\Gamma)\right),
$$

where $\mathcal{O}_{\text {ad }}$ is a set of admissible shape parameters and $\mathcal{O}_{\text {ad }}^{S}$ is the set of admissible boundaries $\Gamma$ such that each $\mathbf{x} \in \mathcal{O}_{\text {ad }}$ determines exactly one $\Gamma \in \mathcal{O}_{\text {ad }}^{S}$. Notice that first-order shape derivatives of the grating efficiency may be computed as

$$
\begin{array}{r}
e_{n}^{\prime}(\Gamma ; a)=\frac{2 k_{y, n}}{k_{y}}\left(\operatorname{Re}\left(\mathcal{F}_{n}(\Gamma)\right) \operatorname{Re}\left(\mathcal{F}_{n}^{\prime}(\Gamma ; a)\right)\right. \\
\left.+\operatorname{Im}\left(\mathcal{F}_{n}(\Gamma)\right) \operatorname{Im}\left(\mathcal{F}_{n}^{\prime}(\Gamma ; a)\right)\right) .
\end{array}
$$

Given (19) and (21), first-order derivatives of the objective function can be computed, requiring the solution of only two integral equations and $2 N$ independent integrals, which may be computed in parallel.

For an initial set-up of the geometry given by $\mathbb{R}^{2 N} \ni \boldsymbol{x}^{(0)}:=\left(\mathcal{A}_{1}^{(0)}, \mathcal{B}_{1}^{(0)}, \ldots, \mathcal{A}_{N}^{(0)}, \mathcal{B}_{N}^{(0)}\right)$, we will consider the usual first-order optimization algorithm (steepest descent):

$$
\boldsymbol{x}^{(t)}=\boldsymbol{x}^{(t-1)}-h^{(t-1)} \nabla f\left(\boldsymbol{x}^{(t-1)}\right),
$$


where $h^{(t-1)}>0$ is the step size of the method at iteration $t$. The step size at each iteration may be found exactly, as

$$
h^{(t-1)}=\arg \min _{h>0} f\left(\boldsymbol{x}^{(t-1)}-h \nabla f\left(\boldsymbol{x}^{(t-1)}\right)\right),
$$

or by employing a backtracking strategy. Since choosing the step size exactly is impractical, we choose to backtrack the step size through the Armijo-Goldstein rule: for a fixed $\alpha \in(0,1), \beta \in\left(0, \frac{1}{2}\right)$ and initial step size estimate $h>0$, we check whether the Armijo-Goldstein condition is satisfied:

$$
f\left(\boldsymbol{x}^{(t-1)}+h \boldsymbol{p}^{t-1}\right)<f\left(\boldsymbol{x}^{(t-1)}\right)-\beta h \boldsymbol{p}^{t-1} \cdot \nabla f\left(\boldsymbol{x}^{(t-1)}\right),
$$

where $\boldsymbol{p}^{(t-1)}=\nabla f\left(\boldsymbol{x}^{(t-1)}\right)$. If (23) holds, then the step size is accepted and $h^{(t-1)}=h$, otherwise the step size estimate is reduced to $\alpha h$. This procedure continues until the step size is accepted. The interested reader may refer to [8, 18, 39, 1] for details.

\subsection{Second-Order Optimization}

First-order optimization algorithms such as the one described in Section 3.3, while being simple and ensuring convergence to local optima, suffer from a number of disadvantages. One of them is their inability to differentiate between local and global optima; this also affects some second-order optimization methods like Newton's method. Moreover, first-order methods commonly need a large number of iterations to achieve convergence and they also cannot differentiate saddle points - points satisfying first-order optimality conditions but not second-order conditions - from local optima. These disadvantages, however, can be eliminated by using second-order methods. Additionally, even if the steepest descent (ascent) algorithm is not exactly at a saddle point (which is unlikely in practice), it behaves poorly when near them, since gradient steps are small in directions that escape saddle points (see [15]).

We can not expect the considered objetive functions to be convex on the design variables; therefore, a modified version of Newton's method for non-convex functions is considered [43, 15]. This method replaces the usual Newton step $\Delta \boldsymbol{x}:=H(\boldsymbol{x})^{-1} \nabla f(\boldsymbol{x})$ with the modified step $\Delta \boldsymbol{x}:=$ $|H(\boldsymbol{x})|^{-1} \nabla f(\boldsymbol{x})$, where $H(\boldsymbol{x})$ is the Hessian matrix of $f(\boldsymbol{x})$, and $|H(\boldsymbol{x})|$ is the matrix resulting from taking the spectral decomposition of $H(\boldsymbol{x})$ and replacing all its negative eigenvalues by their absolute value. The modified Newton iteration is therefore given as

$$
\boldsymbol{x}^{(t)}=\boldsymbol{x}^{(t-1)}-h^{(t-1)}\left|H\left(\boldsymbol{x}^{(t-1)}\right)\right|^{-1} \nabla f\left(\boldsymbol{x}^{(t-1)}\right) .
$$

The modified step not only ensures a faster escape from saddle points than the usual gradient step, but it also yields quadratic convergence in the direction of eigenvectors associated with positive eigenvalues. To make this point clearer, let $Q$ be the matrix with orthonormal eigenvectors of the Hessian matrix $H$ as columns and let $Q_{+}$and $Q_{-}$be the matrices with eigenvectors of $H$ associated to positive and negative eigenvalues, respectively. Consider then the projection of the gradient on the spaces spanned by the columns of $Q_{+}$and $Q_{-}$(hereafter, positive and negative eigenvectors) $\nabla f_{+}$and $\nabla f_{-}$, respectively. The modified Newton step differs from the usual Newton step only in the direction it takes along the space spanned by the negative eigenvectors, since only negative eigenvalues have their sign changed. As such, quadratic convergence along the positive direction $\nabla f_{+}$is expected, while the modification in the negative direction $\nabla f_{-}$allows the method to escape saddle points at an accelerated rate (see Eq. (8) and Theorem 3.2 in [43], as well as the accompanying discussion).

A variant of the algorithm recalculates $|H|$ every $m \in \mathbb{N}$ iterations, i.e.,

$$
\boldsymbol{x}^{(t \cdot m+i)}=\boldsymbol{x}^{(t \cdot m+i-1)}-h^{(t \cdot m+i-1)}\left|H\left(\boldsymbol{x}^{(t \cdot m)}\right)\right|^{-1} \nabla f\left(\boldsymbol{x}^{(t \cdot m+i-1)}\right),
$$


for $i=\{1, \ldots, m\}$ and $k \in \mathbb{N}_{0}$, which proves to be advantageous to reduce the computational burden associated with computing the Hessian matrix and its inverse (see Algorithm 1 in [15]). In our analysis below, both alternatives are implemented and compared. Moreover, the step size for both alternatives is found through backtracking and the Armijo-Goldstein conditions in (23) with $\boldsymbol{p}^{(t-1)}=\left|H\left(\boldsymbol{x}^{(t-1)}\right)\right|^{-1} \nabla f\left(\boldsymbol{x}^{(t-1)}\right)$.

As for first-order derivatives, the adjoint method allows for the approximation of the far-field second derivatives through solving $2 N$ additional integral equations. Accordingly, second-order derivatives for the diffraction efficiency are computed as

$$
\begin{aligned}
& e_{n}^{\prime \prime}\left(\Gamma ; a_{1} ; a_{2}\right)=\frac{2 k_{y, n}}{k_{y}}\left(\operatorname{Re}\left(\mathcal{F}_{n}^{\prime}\left(\Gamma ; a_{1}\right)\right) \operatorname{Re}\left(\mathcal{F}_{n}^{\prime}\left(\Gamma ; a_{2}\right)\right)\right. \\
& +\operatorname{Im}\left(\mathcal{F}_{n}^{\prime}\left(\Gamma ; a_{1}\right)\right) \operatorname{Im}\left(\mathcal{F}_{n}^{\prime}\left(\Gamma ; a_{2}\right)\right)+\operatorname{Re}\left(\mathcal{F}_{n}(\Gamma)\right) \operatorname{Re}\left(\mathcal{F}_{n}^{\prime \prime}\left(\Gamma ; a_{1} ; a_{2}\right)\right) \\
& \left.+\operatorname{Im}\left(\mathcal{F}_{n}(\Gamma)\right) \operatorname{Im}\left(\mathcal{F}_{n}^{\prime \prime}\left(\Gamma ; a_{1} ; a_{2}\right)\right)\right) .
\end{aligned}
$$

Though not reported here in detail, both approaches for computing the first and second-order derivatives of the far-field and the efficiency were validated by comparing them to a finite difference approach.

\subsection{A Quasi-Newton Method: The BFGS Algorithm}

Finally, we consider another variation on Newton's method in the form of a quasi-Newton update (see Chapter 2.6 in [59] for other variations) and compare its performance to both steepest descent and modified Newton methods. The BFGS algorithm - originally developed in [9, 21, 23, 52]employs the following iteration for the minimization of $f(\boldsymbol{x})$ :

$$
\boldsymbol{x}^{(t)}=\boldsymbol{x}^{(t-1)}-h^{(t-1)} B^{(t-1)} \nabla f\left(\boldsymbol{x}^{(t-1)}\right),
$$

where the matrix $B^{(t)}$ is obtained from $B^{(t-1)}$ as

$$
B^{(t)}=B^{(t-1)}-\frac{B^{(t-1)} s^{(t-1)} s^{(t-1)^{\top}} B^{(t-1)}}{s^{(t-1)^{\top}} B^{(t-1)} s^{(t-1)}}-\frac{y^{(t-1)} y^{(t-1)^{\top}}}{s^{(t-1)^{\top}} y^{(t-1)}}
$$

and

$$
\begin{aligned}
& s^{(t-1)}=h^{(t-1)} B^{(t-1)} \nabla f\left(\boldsymbol{x}^{(t-1)}\right), \\
& y^{(t-1)}=\nabla f\left(\boldsymbol{x}^{(t)}\right)-\nabla f\left(\boldsymbol{x}^{(t-1)}\right) .
\end{aligned}
$$

The matrix $B^{(t)}$ must be positive definite and symmetric for all iterations. This condition is guaranteed if the matrix $B^{(0)}$ is initialized as such and each step size $h^{(t)}$ is chosen so that Wolfe conditions are satisfied, i.e., (23) with $\boldsymbol{p}^{(t)}=B^{(t)} \nabla f\left(\boldsymbol{x}^{(t)}\right)$ and

$$
\boldsymbol{p}^{(t-1)} \cdot \nabla f\left(\boldsymbol{x}^{(t-1)}-h^{(t-1)} \boldsymbol{p}^{(t-1)}\right) \leq \gamma \boldsymbol{p}^{(t-1)} \cdot \nabla f\left(\boldsymbol{x}^{(t-1)}\right),
$$

for $\beta \leq \gamma<1$. Though convergence of the algorithm for convex functions is guaranteed, the algorithm, with inexact (Wolfe) line search of the step size, may not converge for non-convex functions (see [14] and references within). 


\section{Numerical Examples}

We present several examples comparing the proposed optimization methods. A standard Galerkin formulation with piecewise polynomials of degree one (previously used in [55]) was employed to numerically solve the required BIEs. All computations were performed on a AMD Opteron 6386 SE server, where parallelization was only used to assemble the relevant matrices.

The given examples deal with the following optimization problems:

$$
\min _{\Gamma}\left(e_{n}(\Gamma)-e_{n}^{\mathrm{obj}}\right) \text { and } \max _{\Gamma} e_{n}(\Gamma),
$$

i.e., we seek for a grating geometry that either attains a certain objective diffraction efficiency or maximizes the diffraction efficiency on a given diffraction mode given wave numbers and incidence angles.

The grating period and its relation to the wavelength also plays an important role. Therefore, it may be chosen according to a desired application, for example, considering the Littrow configuration (as considered in Section 4.3) or second Bragg incidence. After presenting these examples, we compare the performances of the different considered algorithms and, finally, focus on a practical example.

\subsection{Attaining a Specified Diffraction Efficiency}

We start by minimizing an objective function of the form

$$
\tilde{f}\left(e_{n}(\Gamma)\right)=\left(e_{n}(\Gamma)-e_{n}^{\mathrm{obj}}\right)^{2},
$$

for different $n \in \mathbb{N}$ and $e_{n}^{\text {obj }} \in[0,1]$. Convergence of this objective function and design variables are displayed in Figs. 2. Here, a step size $h=1$ was suitable for our modified Newton method with no backtracking of $h$ required. We take a number of steps of gradient descent from a randomized initial geometry (with a fixed small step) and present examples for which the algorithms find optimal geometries, $\Gamma_{\mathrm{obj}}$, such that

$$
\tilde{f}\left(e_{n}\left(\Gamma_{\text {obj }}\right)\right)=0 .
$$

Examples when this fails to happen behave similarly as those presented in the following subsection. Specifically, if the initial efficiency is lower than the objective efficiency and the algorithm fails to find a geometry $\Gamma$ such that $e_{n}(\Gamma)=e_{n}^{\text {obj }}$, the minimization of $\left(e_{n}(\Gamma)-e_{n}^{\text {obj }}\right)^{2}$ behaves like the maximization of $e_{n}(\Gamma)$.

The examples here consider optimization for a previously defined efficiency as required in applications such as hyperspectral imaging, where some practical parameters yield a required diffraction efficiency [50]. Our first example optimizes the $n=-1$ diffraction order to an objective efficiency of $60 \%$ for an incident angle of $\theta_{i}=\pi / 6$ and wavenumber $k=30$. The second example optimizes the $n=1$ diffraction order to an objective efficiency of $65 \%$ for an incident angle of $\theta_{i}=\pi / 4$ and wavenumber $k=40$. The specified incident angles (different than normal incidence) and wavelengths (smaller than the grating period, $\lambda / \Lambda \leq 0.2$ ) were chosen to show the robustness of the method for scattering problems. For the step size backtracking, the chosen parameters were $\alpha=0.5$ and $\beta=0.2$ for all algorithms to ensure a fair comparison. For the Wolfe line search in (27), we chose $\gamma=0.8$.

As indicated in Fig. 2, all implementations of the modified Newton method and the BFGS algorithm — with different initializations for $B^{(0)}$ —appear to be superior in rate to the standard 


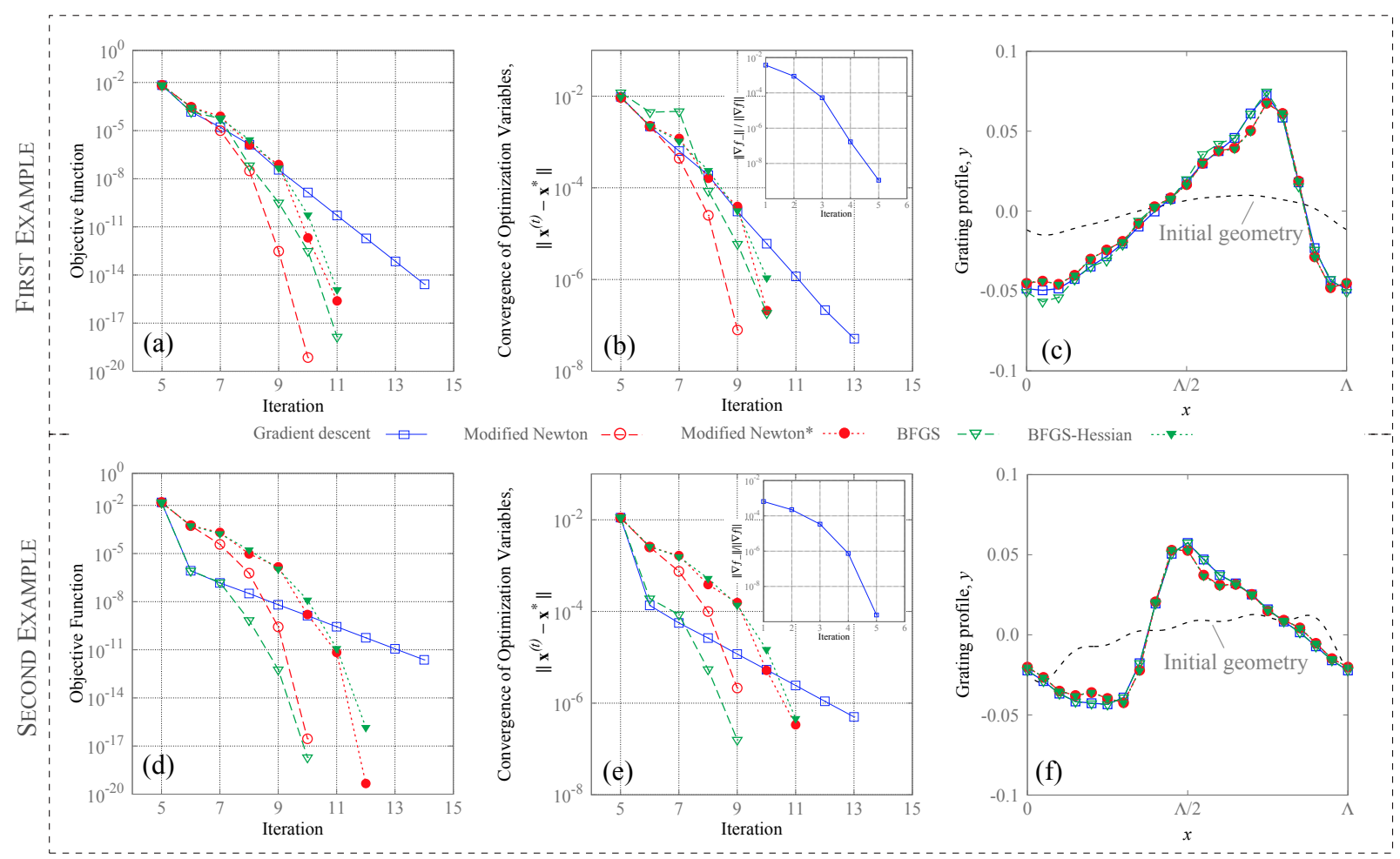

Figure 2: Objective function, error of optimization variables, and optimal geometries are shown considering two minimization examples. Top row corresponds to the grating profile optimization for the diffraction efficiency in the $n=-1$ diffraction order to a target efficiency of $60 \%$ for an incidence angle of $\theta_{i}=\pi / 6$, and wavenumber $k=40$; the bottom row of figures corresponds to the optimization of the diffraction efficiency in the $n=1$ diffraction order to an objective efficiency of $65 \%$ for an incidence angle of $\theta_{i}=\pi / 4$, and wavenumber $k=30$.

The algorithms being compared are specified in the label, where the gradient descent algorithm corresponds to (22), the "Modified Newton" and "Modified Newton*" labels correspond to the algorithms in (24) and (25) with $m=2$, respectively, and the "BFGS" and "BFGS-Hessian" labels correspond to the BFGS algorithm with different initializations for $B^{(0)}$ in $(26)-B^{(0)}=$ Id (the identity matrix) and $B^{(0)}=|H|$, respectively. Only the steps taken before the initial five iterations of gradient descent are shown. For this example, 10 optimization variables were considered $(N=5)$.

gradient descent algorithm. The convergence rate, $q$, of the series of steps $\left\{\boldsymbol{x}^{t}\right\}_{t \in \mathbb{N}}$ may be estimated through the formula

$$
q \approx \log \left(\frac{\left\|\boldsymbol{x}^{(t+3)}-\boldsymbol{x}^{(t+2)}\right\|}{\left\|\boldsymbol{x}^{(t+2)}-\boldsymbol{x}^{(t+1)}\right\|}\right)\left(\log \left(\frac{\left\|\boldsymbol{x}^{(t+2)}-x^{(t+1)}\right\|}{\left\|\boldsymbol{x}^{(t+1)}-\boldsymbol{x}^{(t)}\right\|}\right)\right)^{-1} .
$$

This formula yields an expected convergence rate of $q=1$ for gradient descent, quadratic convergence for the modified Newton algorithm $(q \approx 2)$ and super-linear convergence for the BFGS algorithm $(1<q<2)$.

Furthermore, in both examples, the gradient descent algorithm required to solve (10) a larger number of times per iteration than either the modified Newton algorithm or BFGS (on both implementations of each algorithm) to find a step size satisfying the corresponding conditions. Indeed, the 
modified Newton algorithm and the BFGS algorithm with $B^{(0)}=|H|$ required no backtracking of the step size, while the BFGS algorithm with $B^{(0)}=\mathrm{Id}$ (the identity matrix) required backtracking of the step size.

Quadratic convergence for the modified Newton algorithm and super-linear convergence of the BFGS algorithm are only ensured for convex objective functions. To explain the observed rates of convergence, we examine $\nabla f_{+}$and $\nabla f_{-}$. The insets in Figs. 2(b)-(e) display the convergence to zero of $\left\|\nabla f_{-}\right\| /\|\nabla f\|$, representing the fraction of the gradient $\nabla f$ pointing in directions that oppose quadratic descent. Thus, the contribution of $\nabla f_{-}$is negligible compared to that of $\nabla f_{+}$, which accounts for the seemingly convex behavior of the objective function near its minimum (see [43]).

\subsection{Efficiency Maximization}

We now focus on maximizing objective functions of the form

$$
\tilde{f}\left(e_{n}(\Gamma)\right)=e_{n}(\Gamma)
$$

for different $n \in \mathbb{N}$ and compare, as before, two different examples.

From [43, 15], we expect the iteration count of the modified Newton method to be lower than that for the first-order method, as observed in the previous example. As before, we compare two versions of the modified Newton method (those in (24) and (25) with $m=2$ ) to the firstorder method and two instances of the BFGS algorithm with different initializations for $B^{(0)}$. The algorithms stop if either the gradient of the objective function or the step size at any given iteration fall below a certain tolerance.

Figures 3 and 4 display the diffraction efficiency, at each iteration, of the target mode being optimized for all three methods, the time each method took to arrive at a tolerance $\varepsilon_{t o l}=10^{-2}$ from the maximum efficiency attained, and the final optimized geometries. In general, more than one computation of the objective function was required for the step size backtracking, so the time each method takes to achieve its optimum is not strictly proportional to the number of iterations.

Both versions of the modified Newton method maximize the objective function in fewer iterations and time than gradient ascent, as expected, while also converging more rapidly than the BFGS algorithm. We note that, in this case, neither quadratic nor super-linear convergence was observed.

\subsection{Application: Grating Design}

In this section, gratings are designed and optimized considering the modified Newton algorithm described above. Two grating profiles given by the linear combination of five sines and cosines $(N=5)$ with different initial conditions are considered and the diffraction efficiency on the firstorder mode $(n=1)$ is maximized for a wavelength $\lambda_{0}=300 \mathrm{~nm}$.

For high diffraction efficiency, echelle gratings are commonly used. For this reason, designed gratings are compared to an echelle grating with a blazed angle of $5.2^{\circ}$ in a Littrow configuration $\left(\theta_{i n c}=\theta_{n=1}\right)$. Accordingly, through the grating equation, a period of $\Lambda=1667 \mathrm{~nm}$ is considered for the designed gratings, as well as for the echelle grating.

Though a server was used to obtain all previously presented results at an enhanced level of precision, the studied algorithms may be executed on regular laptops. To demonstrate this, the proposed designs displayed on this section were obtained by running the modified Newton algorithm on a laptop equipped with an Intel(R) Core(TM) i5-3210M CPU and 8 GB DDR3 ram memory, taking no longer than 10 minutes to converge. 

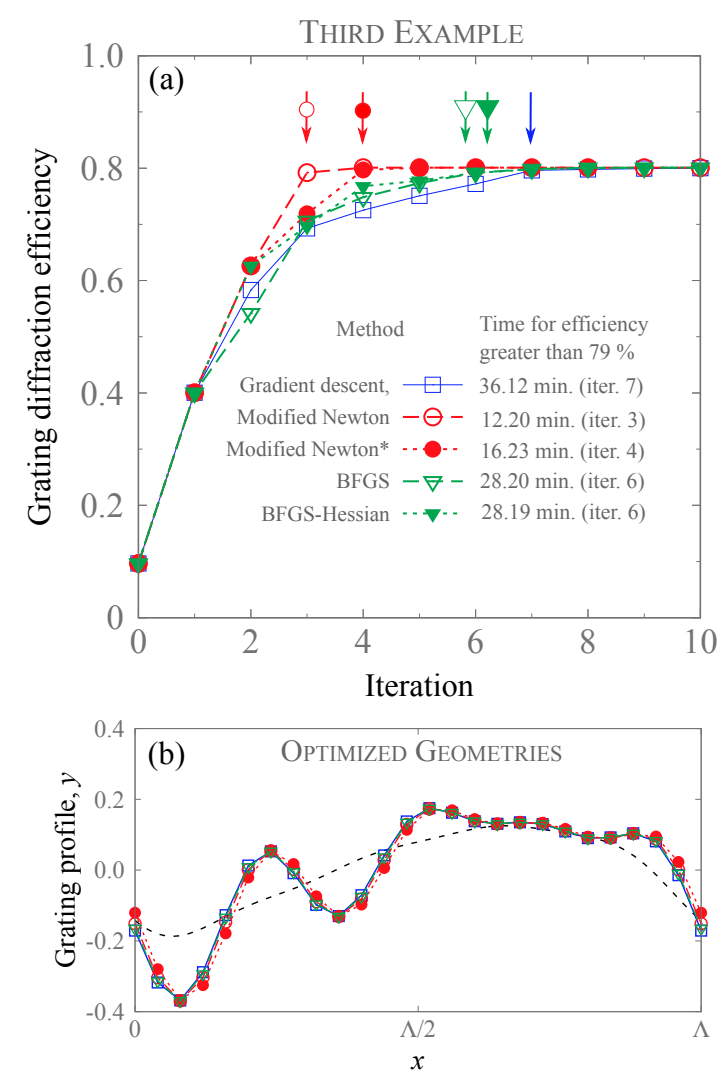

Figure 3: (a) Grating diffraction maximization and (b) optimized geometries are shown for all the studied optimization methods. The parameters correspond to an incidence angle of $\theta=\frac{5}{36} \pi$ and wavenumber $k=20$ for the maximization of the diffraction efficiency in the $n=1$ diffraction order, and $N=4$ (i.e., 8 optimization variables).

Figure 5 shows the efficiency around $\lambda_{0}$ for both designs and the echelle grating (Newport 53101R [42]). The first design gives an efficiency higher than the echelle (72.9\%) only close to $\lambda_{0}$, with a marked peak of $87.6 \%$. The second design exhibits higher efficiency almost for the entire plotted wavelength range, with an efficiency of $85.2 \%$ at $\lambda_{0}$. The sensitivity of the designs to variation was also assessed. Varying the grating parameters (independently) by $5 \%$ was found to reduce the efficiency at $\lambda_{0}$ to $84.8 \%$ and $83.4 \%$ (worst case) for the first and second designs, respectively.

\section{Conclusions}

This work analyzed and compared various optimization algorithms to derive optimal shapes of PEC periodic gratings for high diffraction efficiency for TE polarization.

The modified Newton method for non-convex functions proved to: (i) achieve quadratic convergence of the optimization variables for the objective function in (28); and, (ii) converge to optimal geometries in a lower iteration count than first-order methods for the maximization of grating efficiencies, even if second-order convergence rate is not observed. The BFGS algorithm outperformed the gradient descent method for all considered examples, achieving super-linear convergence for the objective function in (28).

Furthermore, the observed convergence rates in the first two numerical examples seem to be a 

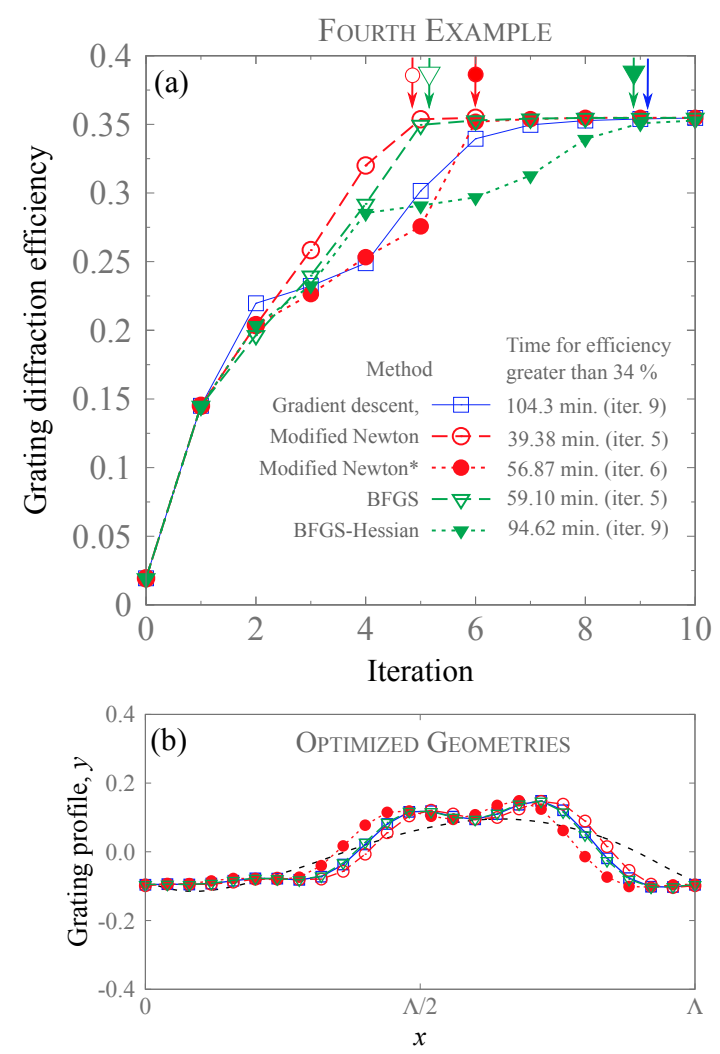

Figure 4: (a) Grating diffraction maximization and (b) optimized geometries are shown for all the studied optimization methods. The parameters correspond to an incidence angle of $\theta=\frac{1}{4} \pi$ and wavenumber $k=30$ for the maximization of the diffraction efficiency in the $n=-1$ diffraction order, and $N=4$ (i.e., 8 optimization variables).

consequence of the convex nature of the objective function in (28) (suggested by the low contribution of $\nabla f_{-}$to $\nabla f$ displayed in the insets of Figs. 2 (b) and (e)), hinting that - when such behavior is expected - the modified Newton method should prove advantageous. In particular, applications of inverse problems employ objective functions that should behave in a similar manner near their optima (see [5] and references therein). Moreover, objective functions similar to those in (28) may prove useful for designing gratings with user-chosen efficiency curves [50].

The variant of the modified Newton method that recomputes $|H|$ every $m>1$ iterations (rather than at each iteration), while increasing the number of steps required to converge, proved to be more efficient than its counterpart $(m=1)$, when high precision is of interest $\left(\varepsilon \leq 10^{-3}\right)$. Both variants of the modified Newton method outperformed gradient descent (ascent) in all examples, while also outperforming the BFGS algorithm on the third and fourth examples.

Finally, two grating profiles were designed using the modified Newton algorithm and compared to a commercial echelle grating. Though both designs differ in their initial geometry, both outperform the commercial grating at the target wavelength, but differ significantly in their behavior at neighboring wavelengths, revealing the role that starting points play.

The results presented throughout demonstrate the applicability of the studied algorithms, paving future research in other settings, such as transmission problems (dielectrics and semiconductors), where different boundary conditions have to be considered. The extension to gratings with corners is also of interest for their applications. However, their optimization presents further 
challenges, mainly because of the computation of shape derivatives in low-regularity profiles.
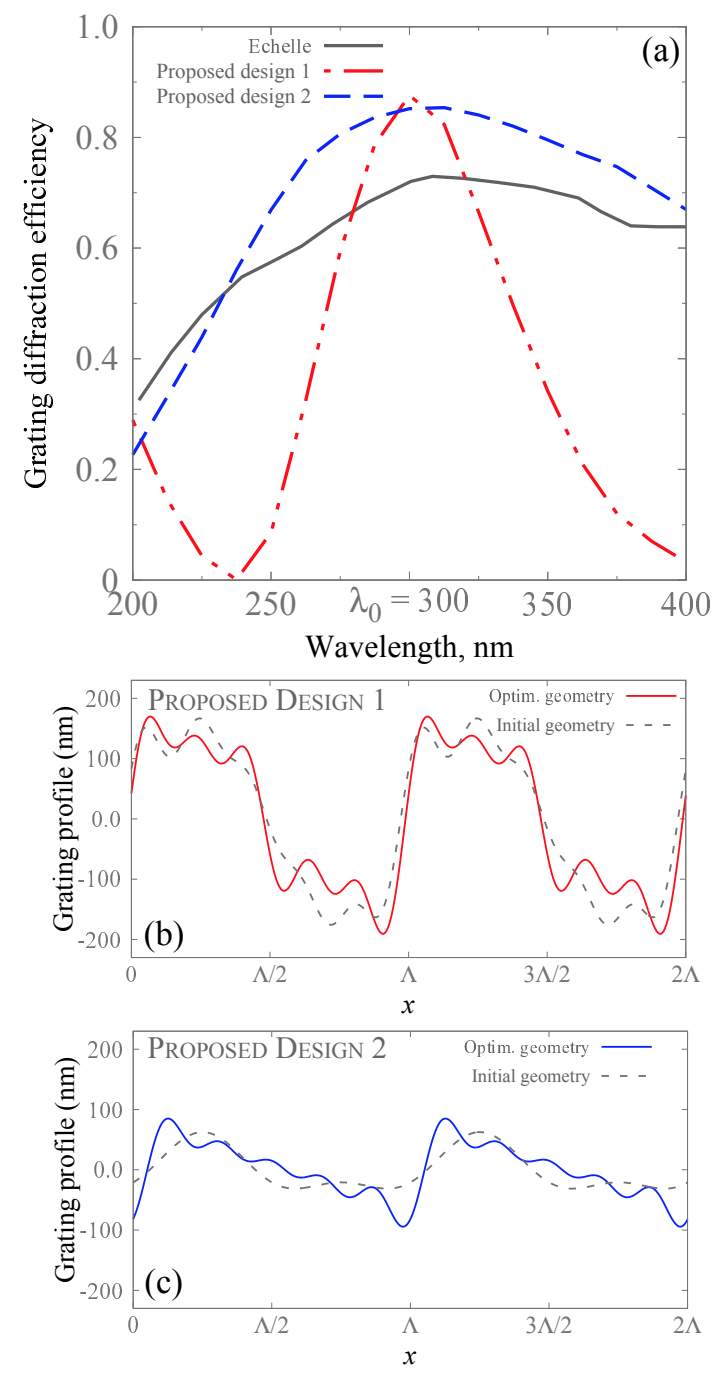

Figure 5: (a) Grating diffraction efficiency for the echelle grating (Newport 53-101R) and two optimized designs, considering 10 optimization variables $(N=5)$. (b) Optimized and initial geometries for the first design and (c) optimized and initial geometries for the second design.

Geometries are optimized for maximal diffraction efficiency on the first-order diffraction mode $(n=1)$ at $\lambda_{0}=300 \mathrm{~nm}$ in the Littrow configuration.

\section{Funding}

This work was sup-ported, in part, by Conicyt-PFCHA/Doctorado Nacional/2017-21171791. C. Jerez-Hanckes work was sponsored by Fondecyt Regular 1171491.

\section{Disclosures}

The authors declare no conflicts of interest. 


\section{References}

[1] Larry Armijo. Minimization of functions having lipschitz continuous first partial derivatives. Pacific Journal of mathematics, 16(1):1-3, 1966.

[2] Mukti Aryal, Doo-Hyun Ko, John R. Tumbleston, Abay Gadisa, Edward T. Samulski, and Rene Lopez. Large area nanofabrication of butterfly wings three dimensional ultrastructures. Journal of Vacuum Science 8 Technology B, Nanotechnology and Microelectronics: Materials, Processing, Measurement, and Phenomena, 30(6):061802, November 2012.

[3] Rubén Aylwin, Carlos Jerez-Hanckes, and José Pinto. On the properties of quasi-periodic boundary integral operators for the Helmholtz equation. Integral Equations and Operator Theory, 92(2):1-41, 2020.

[4] Mark Andreas Bader, Christoph Kappel, André Selle, Jürgen Ihlemann, Mi Li Ng, and Peter R. Herman. F_2-laser-machined submicrometer gratings in thin dielectric films for resonant grating waveguide applications. Applied Optics, 45(25):6586, September 2006.

[5] Gang Bao and David C Dobson. Modeling and optimal design of diffractive optical structures. Surveys on Mathematics for Industry, 8(1):37-62, 1998.

[6] Gang Bao, Peijun Li, and Junliang Lv. Numerical solution of an inverse diffraction grating problem from phaseless data. Journal of the Optical Society of America A, 30(3):293, February 2013.

[7] Anne-Sophie Bonnet-Bendhia and Felipe Starling. Guided waves by electromagnetic gratings and non-uniqueness examples for the diffraction problem. Mathematical Methods in the Applied Sciences, 17(5):305-338, 1994.

[8] Stephen Boyd and Lieven Vandenberghe. Convex Optimization. Cambridge University Press, USA, 2004.

[9] Charles G Broyden. The convergence of a class of double-rank minimization algorithms: 2. the new algorithm. IMA journal of applied mathematics, 6(3):222-231, 1970.

[10] A. Bunkowski, O. Burmeister, T. Clausnitzer, E.-B. Kley, A. Tünnermann, K. Danzmann, and R. Schnabel. Optical characterization of ultrahigh diffraction efficiency gratings. Appl. Opt., 45(23):5795-5799, Aug 2006.

[11] Simon N. Chandler-Wilde, Chris R. Ross, and B. O. Zhang. Scattering by infinite onedimensional rough surfaces. Proceedings of the Royal Society A: Mathematical, Physical and Engineering Sciences, 455(1990):3767-3787, 1999.

[12] Y. B. Chen and K. H. Tan. The profile optimization of periodic nano-structures for wavelengthselective thermophotovoltaic emitters. International Journal of Heat and Mass Transfer, 53(2324):5542-5551, 2010.

[13] Martin Costabel and Frédérique Le Louër. Shape derivatives of boundary integral operators in electromagnetic scattering. part i: Shape differentiability of pseudo-homogeneous boundary integral operators. Integral Equations and Operator Theory, 72(4):509-535, Apr 2012.

[14] Yu-Hong Dai. Convergence properties of the bfgs algoritm. SIAM Journal on Optimization, 13(3):693-701, 2002. 
[15] Yann N Dauphin, Razvan Pascanu, Caglar Gulcehre, Kyunghyun Cho, Surya Ganguli, and Yoshua Bengio. Identifying and attacking the saddle point problem in high-dimensional nonconvex optimization. In Advances in neural information processing systems, pages 2933-2941, 2014 .

[16] Jeffrey A. Davis, Jingo Adachi, Carlos R. Fernández-Pousa, and Ignacio Moreno. Polarization beam splitters using polarization diffraction gratings. Optics Letters, 26(9):587, May 2001.

[17] Afusat O. Dirisu, Claire F. Gmachl, and Deborah L. Sivco. Sub-wavelength antireflection gratings on quantum cascade lasers. In Carmen Mermelstein and David P. Bour, editors, Novel In-Plane Semiconductor Lasers VI. SPIE, February 2007.

[18] Ross Drummond and Stephen Duncan. Accelerated gradient methods with memory. arXiv preprint arXiv:1805.0907r, 2018.

[19] K. Eppler and H. Harbrecht. Second-order shape optimization using wavelet BEM. Optimization Methods and Software, 21(1):135-153, February 2006.

[20] Karsten Eppler. Boundary integral representations of second derivatives in shape optimization. Discussiones Mathematicae. Differential Inclusions, Control and Optimization, 20(1):63, 2012.

[21] Roger Fletcher. A new approach to variable metric algorithms. The computer journal, 13(3):317-322, 1970.

[22] Wentao Gao, Aibibula Abudula, and Zhijun Sun. Multiplet independent resonance modes in multiplexed gratings. Opt. Express, 27(20):28254-28263, Sep 2019.

[23] Donald Goldfarb. A family of variable-metric methods derived by variational means. Mathematics of computation, 24(109):23-26, 1970.

[24] Noah Graham. Casimir energies of periodic dielectric gratings. Physical Review A - Atomic, Molecular, and Optical Physics, 90(3), 2014.

[25] A E Grigorescu and C W Hagen. Resists for sub-20-nm electron beam lithography with a focus on HSQ: state of the art. Nanotechnology, 20(29):292001, July 2009.

[26] Frank Hettlich and William Rundell. A second degree method for nonlinear inverse problems. SIAM Journal on Numerical Analysis, 37(2):587-620, 1999.

[27] Hiroaki Honma, Masato Mitsudome, Shintaro Itoh, Makoto Ishida, Kazuaki Sawada, and Kazuhiro Takahashi. Fabrication of free-standing subwavelength metal-insulator-metal gratings using high-aspect-ratio nanoimprint techniques. Japanese Journal of Applied Physics, 55(6S1):06GP20, May 2016.

[28] Guanghui Hu and Andreas Rathsfeld. Scattering of time-harmonic electromagnetic plane waves by perfectly conducting diffraction gratings. IMA Journal of Applied Mathematics (Institute of Mathematics and Its Applications), 80(2):508-532, 2015.

[29] Imran Khan, Hamid Keshmiri, Florian Kolb, Theodoros Dimopoulos, Emil J. W. ListKratochvil, and Jakub Dostalek. Multidiffractive broadband plasmonic absorber. Advanced Optical Materials, 4(3):435-443, 2016.

[30] Andreas Kirsch. Diffraction by periodic structures. In Inverse problems in mathematical physics, pages 87-102. Springer, 1993. 
[31] ANDREAS Kirsch. The domain derivative and two applications in inverse scattering theory. Inverse problems, 9(1):81-96, 1993.

[32] Nolan Lassaline, Raphael Brechbühler, Sander J. W. Vonk, Korneel Ridderbeek, Martin Spieser, Samuel Bisig, Boris le Feber, Freddy T. Rabouw, and David J. Norris. Optical fourier surfaces. Nature, 582(7813):506-510, 2020.

[33] Yuzhang Liang, Wei Peng, Rui Hu, and Helin Zou. Extraordinary optical transmission based on subwavelength metallic grating with ellipse walls. Optics Express, 21(5):6139, 2013.

[34] C. M. Linton. The green's function for the two-dimensional Helmholtz equation in periodic domains. Journal of Engineering Mathematics, 33(4):377-401, May 1998.

[35] C. Lu and R.H. Lipson. Interference lithography: a powerful tool for fabricating periodic structures. Laser 8 Photonics Reviews, 4(4):568-580, May 2009.

[36] A A Maradudin, I Simonsen, J Polanco, and R M Fitzgerald. Rayleigh and wood anomalies in the diffraction of light from a perfectly conducting reflection grating. Journal of Optics, 18(2):024004, January 2016.

[37] Randall McEntaffer, Casey DeRoo, Ted Schultz, Brennan Gantner, James Tutt, Andrew Holland, Stephen O'Dell, Jessica Gaskin, Jeffrey Kolodziejczak, William W. Zhang, Kai-Wing Chan, Michael Biskach, Ryan McClelland, Dmitri Iazikov, Xinpeng Wang, and Larry Koecher. First results from a next-generation off-plane x-ray diffraction grating. Experimental Astronomy, 36(1-2):389-405, May 2013.

[38] Jean-Claude Nédélec and Felipe Starling. Integral equation methods in a quasi-periodic diffraction problem for the time-harmonic maxwell's equations. SIAM Journal on Mathematical Analysis, 22(6):1679-1701, 1991.

[39] Yurii Nesterov. Lectures on convex optimization, volume 137. Springer, 2018.

[40] Manoj Niraula, Jae Woong Yoon, and Robert Magnusson. Mode-coupling mechanisms of resonant transmission filters. Optics Express, 22(21):25817, October 2014.

[41] A. Paganini, S. Sargheini, R. Hiptmair, and Ch. Hafner. Shape optimization of microlenses. Optics Express, 23(10):13099, 2015.

[42] Christopher Palmer and Erwin Loewen. Diffraction Gratings Handbook, volume 1. 2014.

[43] Santiago Paternain, Aryan Mokhtari, and Alejandro Ribeiro. A newton-based method for nonconvex optimization with fast evasion of saddle points. SIAM Journal on Optimization, 29(1):343-368, January 2019.

[44] Roger Petit. Electromagnetic Theory of Gratings. Springer Berlin Heidelberg, 1980.

[45] Oliver Pironneau, editor. Optimal Shape Design for Elliptic Systems. Springer Berlin Heidelberg, 1984.

[46] Giorgio Quaranta, Guillaume Basset, Olivier J. F. Martin, and Benjamin Gallinet. Recent advances in resonant waveguide gratings. Laser $\mathcal{E}$ Photonics Reviews, 12(9):1800017, July 2018 . 
[47] L. Rayleigh. On the dynamical theory of gratings. Proceedings of the Royal Society A: Mathematical, Physical and Engineering Sciences, 79(532):399-416, August 1907.

[48] A Roger. Generalized reciprocity relations for perfectly conducting gratings. Optica Acta: International Journal of Optics, 29(10):1427-1439, 1982.

[49] A. Roger. Optimization of perfectly conducting gratings a general method. Optica Acta: International Journal of Optics, 30(3):387-398, March 1983.

[50] Bernard Sabushimike, Georges Horugavye, and Serge Habraken. Optimization of a multiblaze grating in reflection using a free-form profile. Applied optics, 57(18):5048-5056, 2018.

[51] Muhammad Rizwan Saleem, Rizwan Ali, Mohammad Bilal Khan, Seppo Honkanen, and Jari Turunen. Impact of atomic layer deposition to nanophotonic structures and devices. Frontiers in Materials, 1, October 2014.

[52] David F Shanno. Conditioning of quasi-newton methods for function minimization. Mathematics of computation, 24(111):647-656, 1970.

[53] Mehrdad Shokooh-Saremi and Robert Magnusson. Particle swarm optimization and its application to the design of diffraction grating filters. Optics Letters, 32(8):894, March 2007.

[54] G. Silva-Oelker, C. Jerez-Hanckes, and P. Fay. High-temperature tungsten-hafnia optimized selective thermal emitters for thermophotovoltaic applications. Journal of Quantitative Spectroscopy and Radiative Transfer, 231:61-68, July 2019.

[55] Gerardo Silva-Oelker, Ruben Aylwin, Carlos Jerez-Hanckes, and Patrick Fay. Quantifying the impact of random surface perturbations on reflective gratings. IEEE Transactions on Antennas and Propagation, 66(2):838-847, February 2018.

[56] Gerardo Silva-Oelker, Carlos Jerez-Hanckes, and Patrick Fay. Study of w/HfO2 grating selective thermal emitters for thermophotovoltaic applications. Optics Express, 26(22):A929, October 2018.

[57] Jan Sokolowski and Jean-Paul Zolésio. Introduction to shape optimization. In Introduction to Shape Optimization, pages 5-12. Springer, 1992.

[58] Manuel E. Solano, Muhammad Faryad, Peter B. Monk, Thomas E. Mallouk, and Akhlesh Lakhtakia. Periodically multilayered planar optical concentrator for photovoltaic solar cells. Applied Physics Letters, 103(19):191115, November 2013.

[59] Gilbert Strang. Computational Science and Engineering. Wellesley-Cambridge, 2007.

[60] Sudheer, S. Porwal, S. Bhartiya, B. T. Rao, P. Tiwari, Himanshu Srivastava, T. K. Sharma, V. N. Rai, A. K. Srivastava, and P. A. Naik. Diffraction efficiency of plasmonic gratings fabricated by electron beam lithography using a silver halide film. Journal of Applied Physics, 120(4):043101, July 2016.

[61] Leung Tsang, Jin Au Kong, Kung-Hau Ding, and Chi On Ao. Scattering of Electromagnetic Waves: Numerical Simulations. John Wiley \& Sons, Inc., May 2001.

[62] Joan Vila-Comamala, Lucia Romano, Vitaliy Guzenko, Matias Kagias, Marco Stampanoni, and Konstantins Jefimovs. Towards sub-micrometer high aspect ratio x-ray gratings by atomic layer deposition of iridium. Microelectronic Engineering, 192:19-24, May 2018. 
[63] Kang Wei and Yi Zhao. Fabrication of anisotropic and hierarchical undulations by benchtop surface wrinkling. In 2014 IEEE 27th International Conference on Micro Electro Mechanical Systems (MEMS). IEEE, January 2014.

[64] R W Wood. On a remarkable case of uneven distribution of light in a diffraction grating spectrum. Proceedings of the Physical Society of London, 18(1):269-275, June 1902.

[65] Zhaoning Yu, Lei Chen, Wei Wu, Haixiong Ge, and Stephen Y. Chou. Fabrication of nanoscale gratings with reduced line edge roughness using nanoimprint lithography. Journal of Vacuum Science $\&$ Technology B: Microelectronics and Nanometer Structures, 21(5):2089, 2003.

[66] Uwe D. Zeitner, Maria Oliva, Frank Fuchs, Dirk Michaelis, Tino Benkenstein, Torsten Harzendorf, and Ernst-Bernhard Kley. High performance diffraction gratings made by e-beam lithography. Applied Physics A, 109(4):789-796, October 2012.

[67] Bo Zhang and Simon N. Chandler-Wilde. Integral equation methods for scattering by infinite rough surfaces. Mathematical Methods in the Applied Sciences, 26(6):463-488, 2003. 